\title{
OPTICS SIMULATIONS FOR THE NLC MAIN LINAC*
}

\author{
M. Woodley ${ }^{\dagger}$, P. Tenenbaum, L. Hendrickson, A. Seryi, \\ SLAC, Stanford, CA 94309, USA
}

\begin{abstract}
Focusing in the NLC main linac will be provided mainly by hybrid permanent magnet quadrupoles which have limited variability in strength. When the energy profile of the linac changes, due to normal cycling of $\mathrm{rf}$ sources, mismatches in the beam optics can be generated if the quadrupole strengths are not rescaled to the new energy profile. These mismatches can lead to emittance dilution. In addition, betatron phase advance changes caused by the mismatch can adversely affect the beam trajectory, leading to emittance dilution from dispersion and wakefields. This paper describes the results of simulations of these processes, undertaken in an attempt to determine whether or not rescaling of the quadrupoles will be necessary in the NLC main linac.
\end{abstract}

\section{INTRODUCTION}

The NLC main linacs are designed to accelerate $8 \mathrm{GeV}$ electron or positron beams to $250 \mathrm{GeV}$. The acceleration takes place in $0.9 \mathrm{~m}$ long $\mathrm{X}$-band $(11.4 \mathrm{GHz})$ accelerating structures at an effective gradient of $48 \mathrm{MV} / \mathrm{m}$. For $\mathrm{rf}$ distribution purposes each linac is divided into 13 sectors, plus two diagnostic regions. Each sector is $468.6 \mathrm{~m}$ long and contains 9 interleaved $\mathrm{rf}$ units. Each $\mathrm{rf}$ unit is composed of a modulator powering 8 pulsed klystrons (an "8-pack") and a Delay Line Distribution System (DLDS) which delivers the summed rf output of the klystrons to the structures [1]. Each linac contains 117 8-packs. During normal operations, approximately $5 \%$ of these will be held in reserve as available spares. Figure 1 shows a schematic of the rf power distribution for a single linac sector.

\section{LINAC SECTOR}

Delay Line Distribution System (DLDS) NONET

$9 \times 24$ Accelerator Structures

Active $/$ Actual Length $=9 \times 24 \times 1.8 / 450=86.3 \%$

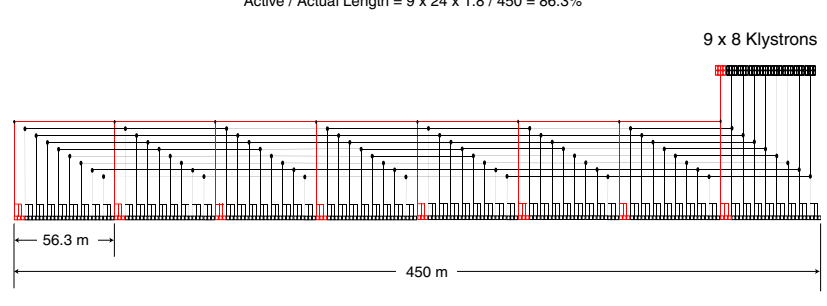

Figure 1: Schematic of rf power distribution from 8-packs to X-band structures for a typical NLC main linac sector.

\footnotetext{
*Work supported by the U.S. Department of energy, Contract DE-AC03-76SF00515.

†Email: mdw@slac.stanford.edu
}

During normal operations, klystrons will periodically switch off for a variety reasons. Although most of these faults will be of relatively short duration (a few machine pulses at $120 \mathrm{~Hz}$ ), some faulted klystrons will not recover without intervention. When an individual klystron faults, its respective 8-pack is automatically deactivated to protect the faulted klystron from damage. If the fault doesn't reset immediately, a spare 8-pack which has been held in reserve will be activated in order to make up the energy deficit. Although the total beam energy at the end of the linac is maintained under these circumstances, the energy profile along the linac is changed because the distribution of powered and unpowered structures has changed.

Experience with the SLAC linac [2] has shown that energy profile changes, if not compensated, can lead to emittance growth. The local mismatch of the quadrupole strengths to the energy profile causes a beta mismatch which can generate emittance dilution by phase mixing (filamentation). More importantly, the betatron phase advance changes in the region of the mismatch, degrading the trajectory of the beam in a previously steered machine and leading to emittance dilution due to dispersion and wakefields.

In this paper we will investigate the consequences of energy profile changes on the NLC beam emittance by simulating the process of 8-pack exchange.

\section{MAD SIMULATION}

The MAD program [3] is used to simulate the effect on the linac beta functions of exchanging 8-packs. This effect is quantified by the beta-mismatch parameter, BMAG [4], defined in terms of Twiss parameters as:

$$
\mathrm{BMAG}=\frac{1}{2}(\beta \tilde{\gamma}-2 \alpha \tilde{\alpha}+\gamma \tilde{\beta}) \geq 1
$$

where the values with and without a " $\sim$ " correspond to the mismatched and matched lattice, respectively. The mismatch of the beam shape to the transport lattice causes filamentation of phase space because of the finite energy spread of the bunch. In a long FODO array like the linac, filamentation due to the beta mismatch leads to emittance dilution proportional to BMAG.

The ideal, matched NLC linac, with no errors, is first simulated. The nominal energy profile includes BNS phasing to reduce single-bunch beam breakup (BBU) effects [5]. Figure 2 shows the beam energy, BNS rf phase, and BNS energy spread along the linac for a nominal NLC bunch of $0.75 \times 10^{10}$ particles with an initial rms bunch length and energy spread of $110 \mu \mathrm{m}$ and $1.6 \%$, respectively. Six 8-packs are held in reserve (spares): one 
for each of the three BNS regions, and one for each energy feedback (immediately upstream of each diagnostic region and one at the end of the linac).
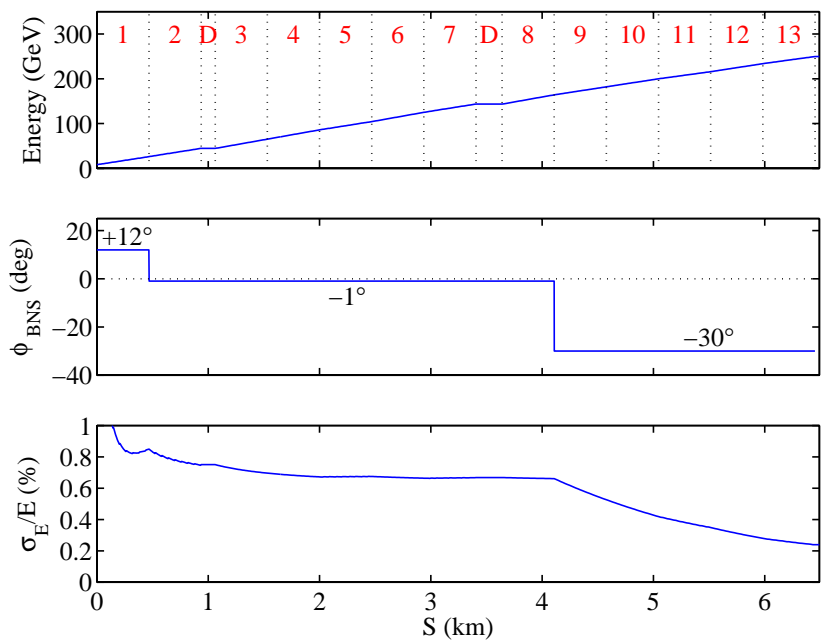

Figure 2: Beam energy (top), BNS rf phase (middle), and BNS energy spread (bottom), along the NLC main linac. Linac sectors and diagnostic regions are labeled on the upper plot in red.
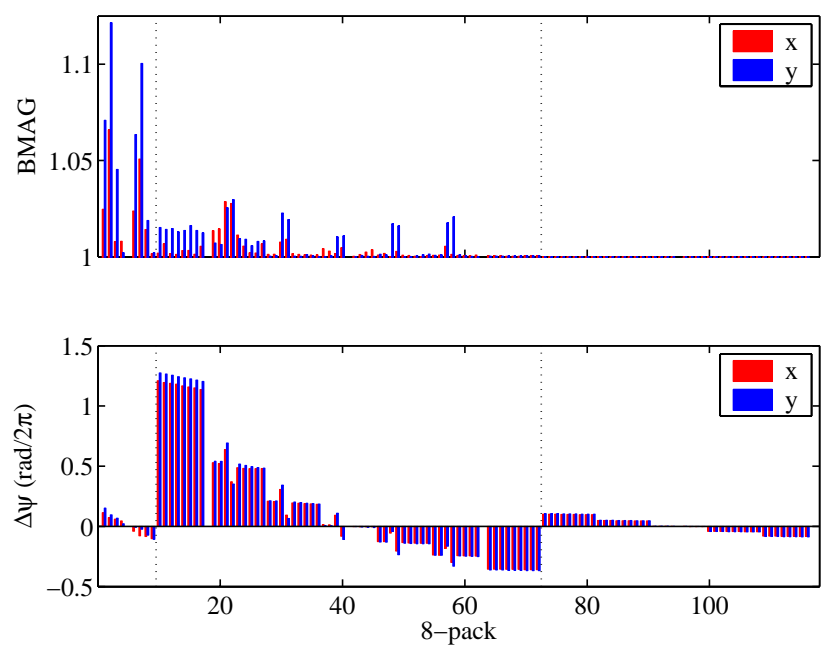

Figure 3: BMAG (upper) and betatron phase advance change (lower) at the end of the NLC main linac versus exchanged 8-pack. BNS region boundaries are indicated by vertical dotted lines.

Next, 8-packs are turned off one by one in order along the linac. An 8-pack which is turned off in a given BNS region is replaced by the designated spare 8-pack for that region, thus keeping the final energy constant. The designated spare 8-pack for each BNS region is located near the middle of the region. The magnetic fields of the quadrupoles are held constant, and MAD is used to compute the Twiss parameters of the resultant mismatched machine. Figure 3 summarizes the simulation results. The upper plots show BMAG at the end of the linac versus exchanged 8-pack number. The lower plots show the net change in betatron phase advance at the end of the linac. The maximum values of both BMAG and phase shift correspond to exchanging 8-packs near the beginning of the linac, where the fractional change to the beam energy is largest.

\section{LIAR SIMULATION}

The LIAR program [6] is used to simulate the effect of 8-pack exchange on the linac trajectory. Only effects in the vertical plane are considered since the vertical emittance of the NLC beam is only $1 \%$ of the horizontal and therefore much more sensitive to dilution effects. The nominal NLC linac is set up in LIAR and misaligned using a standard set of misalignment parameters, summarized in Table 1.

Table 1: Misalignment Parameters

\begin{tabular}{|l|c|c|}
\hline Element & $\Delta \mathbf{Y ~ r m s}$ & comment \\
\hline rf structures & $15 \mu \mathrm{m}$ & \\
\hline rf girders & $50 \mu \mathrm{m}$ & BPMs in quads move with \\
quadrupoles & $50 \mu \mathrm{m}$ & $\begin{array}{c}\text { BPM-to-quad-center } \\
\text { residual after Beam Based } \\
\text { Alignment }\end{array}$ \\
\hline BPMs & $2 \mu \mathrm{m}$ & \begin{tabular}{c} 
Alignen \\
\hline
\end{tabular} \\
\hline
\end{tabular}

LIAR is then used to steer the beam using quadrupole movers and rf girder movers [7]. Finally, an 8-pack is exchanged and the effect on both the beam trajectory and the emittance are observed. This procedure is repeated 10 times, using different random seeds, for each 8-pack exchange. Figure 4 summarizes the results of the simulations. The upper plot shows the rms over the entire linac of the residual orbit after each 8-pack exchange. The lower plot shows the emittance dilution at the end of the linac.
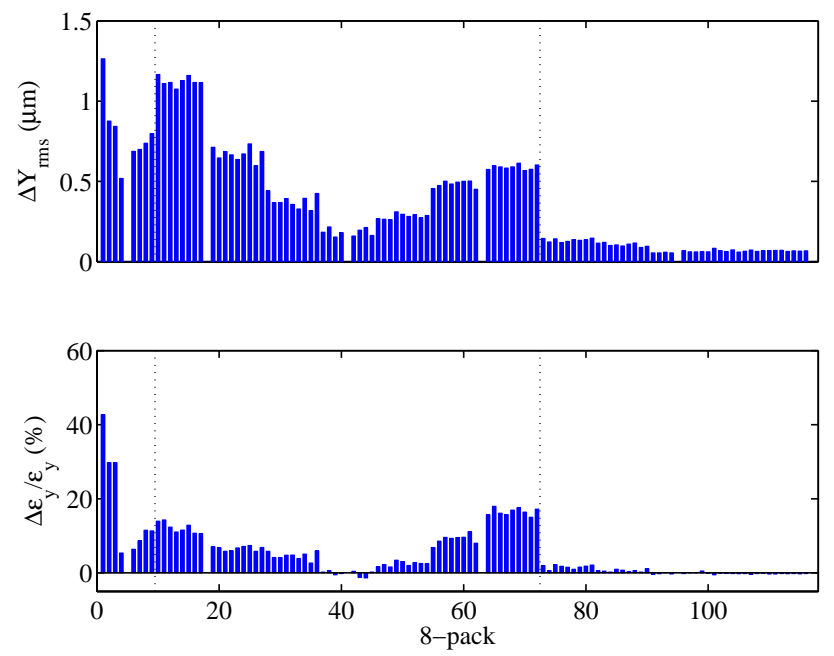

Figure 4: Vertical orbit rms over entire NLC main linac, after misalignment, correction, and 8-pack exchange, versus exchanged 8-pack (top); relative emittance growth at end of linac versus exchanged 8-pack (bottom). 


\section{DISCUSSION}

The emittance dilution expected from the BMAG effects shown in Figure 3 is, for most of the linac, only a few percent at most and is not considered a problem. This is due to the fact that the acceleration due to any single 8pack is interleaved within a sector with that of the other eight 8-packs of the sector. This is shown in Figure 1, where power flow from the first 8-pack is shown in red. Consider the first 8-pack of the linac, where the beam energy is lowest. Its energy gain represents more than $25 \%$ of the injected beam energy of $8 \mathrm{GeV}$. However, the maximum relative energy error incurred by turning the first 8 -pack off is only $3 \%$ because of the interleaving. For the SLAC linac, with no interleaving, turning off the first klystron seen by the $1.2 \mathrm{GeV}$ beam from the damping ring would mean a relative energy error of $20 \%$. For the SLAC linac, scaling of the quadrupole strengths is essential when klystrons are exchanged.

The phase advance change due to the local mismatch of the quadrupole strength to the energy profile causes a breakdown in the steering which engenders emittance dilution at the end of the linac. Figure 4 shows the rms of the residual vertical orbit and the resultant emittance dilution when 8-packs are exchanged. Figure 5 shows the correlation between orbit rms and emittance dilution for each of the BNS regions of the linac. It is clear that if the orbit were recentered after an 8-pack exchange the emittance dilution would be greatly reduced.

\section{CONCLUSIONS}

Dilution of the vertical emittance due to filamentation of the beta mismatch generated by 8 -pack exchange is mitigated by the layout of the rf power flow in the linac, and is considered not to be a reason to require quadrupole scaling. The vertical emittance dilution due to the trajectory effects of 8-pack exchange are more serious and indicate that some action needs to be taken. It appears that if the orbit is recentered then the emittance dilution would likely be acceptable. The design of the NLC main linac includes several trajectory control feedbacks along the linac [8]. These feedbacks would automatically restore the trajectory after any 8-pack exchange. Therefore it appears that scaling of quadrupole strengths will not be necessary. However, this capability will be maintained as part of the design.

Further studies are required, including simulation of the correction of the post-exchange orbit by feedback, and the effects of 8-pack exchange on the use of closed trajectory bumps which may be used correct the emittance.

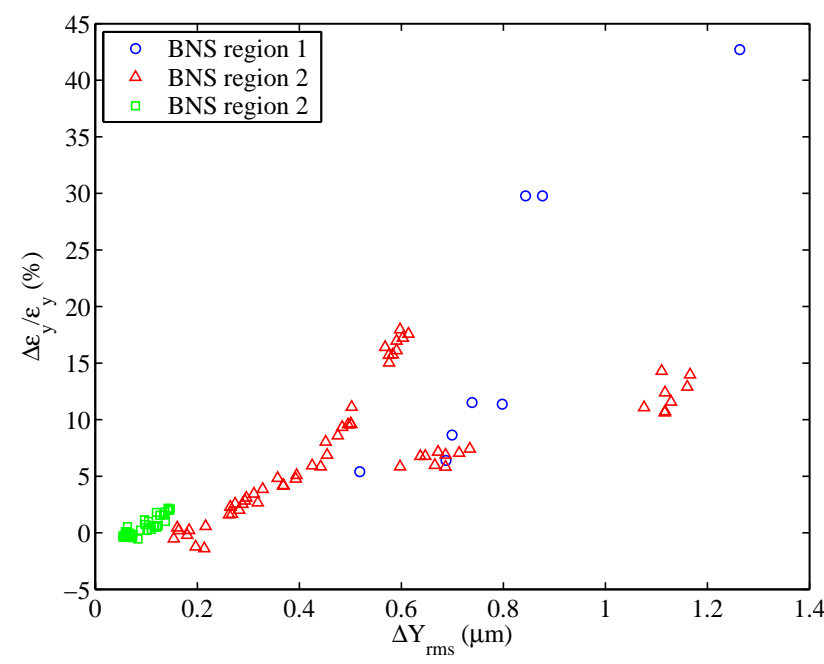

Figure 5: Vertical emittance dilution at the end of the NLC main linac versus the rms vertical orbit due to 8-pack exchange (averaged over 10 seeds).

\section{ACKNOWLEDGEMENTS}

The authors wish to acknowledge the contributions to this work of T. Raubenheimer and C. Adolphsen.

\section{REFERENCES}

[1] N. Phinney, gen.ed., "2001 Report on the Next Linear Collider", SLAC-Report-571 (June 2001).

[2] T. Himel, K. Thompson, "Energy Measurements From Betatron Oscillations", SLAC-PUB-4917 (March 1989).

[3] H. Grote, F.C. Iselin, "The MAD Program User's Reference Manual”, CERN/SL/90-13 (AP) (Rev. 5) (April 1996).

[4] F.J. Decker, et al, "Dispersion and Betatron Matching into the Linac", SLAC-PUB-5484 (May 1991).

[5] http://www-project.slac.stanford.edu/lc/ilc/ TechNotes/lccnotes/PDF/bns_Stupakov.pdf.

[6] R. Assman, et al, "The Computer Program LIAR for the Simulation and Modeling of High Performance Linacs", SLAC-PUB-7577 (July 1997).

[7] P. Tenenbaum, "Simulation Studies of Main Linac Steering in the Next Linear Collider", SLAC-PUB8138 (March 1999).

[8] L. Hendrickson, et al, "Beam-Based Feedback Simulations for the NLC Linac", SLAC-PUB-8584 (September 2000). 